\title{
State of the art thoracic ultrasound: intervention and therapeutics
}

\author{
John P Corcoran, ${ }^{1,2}$ Rachid Tazi-Mezalek, ${ }^{3}$ Fabien Maldonado, ${ }^{4}$ Lonny B Yarmus, \\ Jouke T Annema, ${ }^{6}$ Coenraad F N Koegelenberg, ${ }^{7,8}$ Victoria St Noble, ${ }^{9}$ \\ Najib M Rahman ${ }^{1,2,10}$
}

${ }^{1}$ Oxford Centre for Respiratory Medicine, Oxford University Hospitals NHS Foundation Trust, Oxford, UK

${ }^{2}$ University of Oxford Respiratory Trials Unit, Churchill Hospital, Oxford, UK

${ }^{3}$ Department of Thoracic Oncology, Pleural Diseases and Interventional Pulmonology, Hôpital Nord, Aix-Marseille University, Marseille, France ${ }^{4}$ Division of Allergy, Pulmonary and Critical Care Medicine Vanderbilt-Ingram Cancer Center, Vanderbilt University School of Medicine, Nashville, Tennessee, USA

${ }^{5}$ Division of Pulmonary and Critical Care Medicine, John Hopkins University, Baltimore, Maryland, USA

${ }^{6}$ Department of Pulmonology, Academic Medical Centre, University of Amsterdam, Amsterdam, The Netherlands ${ }^{7}$ Division of Pulmonology, Department of Medicine, Stellenbosch University, Cape Town, South Africa

${ }^{8}$ Tygerberg Academic Hospital, Cape Town, South Africa

${ }^{9}$ Department of Radiology, Oxford University Hospitals NHS Foundation Trust, Oxford, UK ${ }^{10}$ NIHR Oxford Biomedical Research Centre, University of Oxford, Oxford, UK

\section{Correspondence to}

Dr John P Corcoran, University of Oxford Respiratory Trials Unit Churchill Hospital, Oxford OX3 7LE, UK;

jpcorcoran@doctors.org.uk

Received 23 August 2016 Revised 14 March 2017

Accepted 23 March 2017 Published Online First 13 April 2017

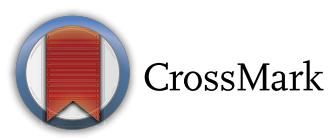

To cite: Corcoran JP, TaziMezalek R, Maldonado F, et al. Thorax

2017:72:840-849.

\section{ABSTRACT}

The use of thoracic ultrasound outside the radiology department and in everyday clinical practice is becoming increasingly common, having been incorporated into standards of care for many specialties. For the majority of practitioners, their experience of, and exposure to, thoracic ultrasound will be in its use as an adjunct to pleural and thoracic interventions, owing to the widely recognised benefits for patient safety and risk reduction. However, as clinicians become increasingly familiar with the capabilities of thoracic ultrasound, new directions for its use are being sought which might enhance practice and patient care. This article reviews the ways in which the advent of thoracic ultrasound is changing the approach to the investigation and treatment of respiratory disease from an interventional perspective. This will include the impact of thoracic ultrasound on areas including patient safety, diagnostic and therapeutic procedures, and outcome prediction; and will also consider potential future research and clinical directions.

\section{INTRODUCTION}

Thoracic ultrasound (TUS) has, in recent years, become as essential a tool as the stethoscope in the management of patients with respiratory disease. There has been a change in attitude among physicians with growing recognition that clinical examination and plain chest radiography are often imprecise. $^{1}{ }^{2}$ This evolution of clinical practice has been facilitated in part by technological progress, with the increasing availability of high-specification portable ultrasound machines that can be easily brought to the patient's bedside. Alongside this, the incorporation of ultrasound training standards into postgraduate medical curricula ${ }^{3-5}$ has led to a new generation of clinicians emerging with a set of clinical skills and interests that mean the use of TUS is continually expanding and evolving.

This revolution is clearly seen in the field of interventional pulmonology, where the advent of TUS has dramatically altered the range of procedures and the way in which they are performed. For many practitioners, TUS may be considered as little more than a means of confirming the presence or absence of pleural fluid before intervention. In this review article we aim to demonstrate how and why this way of thinking is only the beginning; inviting the reader to consider the current capabilities of TUS, alongside the way in which ongoing work is changing the way we investigate and treat patients with respiratory disease. We will examine the practicalities of using TUS in everyday clinical work, and the evidence relevant to patient safety that underpins this. The impact of TUS on basic and more advanced procedures will be described, together with an overview of how outcome prediction and future research will continue to change the way we practise in years to come.

\section{PRACTICAL CONSIDERATIONS}

Clinical practitioners who regularly use TUS must have completed training according to an approved syllabus $^{3-5}$ under appropriate supervision, and maintain a record of practice that is subject to audit (internal or external) and demonstrates continued competence. All practitioners must recognise their own limitations, those of colleagues and of their working environment; ideally, there should be a clear upward referral pathway that allows access to the support of experienced colleagues either in the radiology department or via a multidisciplinary team interaction. The skills and experience a practitioner needs to acquire in the use of TUS will vary according to their specialty and geographical area of practice. Much will depend on what is needed for an individual's everyday clinical responsibilities, and what their workload is likely to allow them to maintain competence in performing.

Conventional brightness (B)-mode ultrasound provides the operator with sufficient information for the majority of thoracic TUS applications and interventions, but most modern machines will offer the operator a selection of other options, including motion (M)-mode and colour flow Doppler scanning. A low-frequency $(2-5 \mathrm{MHz})$ curvilinear probe is the most useful if only one can be selected, allowing both superficial and deep structures to be evaluated as a consequence of the tissue penetration on offer. This is at the expense of image quality, and if depth can be sacrificed (eg, when assessing chest wall and/or parietal pleural disease) then a high-frequency $(5-10 \mathrm{MHz})$ linear probe will provide additional detail and resolution. If default or preset ultrasound settings are offered by the machine, these should be chosen to suit the work being done-'abdominal' mode will serve for interventions where depth of view is important (eg, assessment of pleural effusions and distal structures, such as lung and mediastinum); whereas 'thyroid' or 'musculoskeletal' mode should be selected for proximal work dealing with the chest wall or superficial lymph nodes. In many cases, further optimisation of image quality can be achieved by spending time at the start of any ultrasound assessment 
adjusting parameters, such as gain, depth and time-gain compensation - the success of such fine tuning will depend on the experience of the operator and their familiarity with the characteristics of a specific machine.

Achieving a safe and controlled environment for intervention, including optimal patient positioning, is important yet often underappreciated. Many practitioners will be familiar with emergency 'point of care' diagnostic TUS in the acutely unwell patient, where scanning is unpredictable and performed at all hours, usually by a single bedside operator with the patient in a supine position and using a zonal approach. ${ }^{6}$ By contrast, interventional TUS is largely used in an elective theatre or clean room setting by a clinical team where consideration can be given to the patient's position according to the area of potential interest, determined in advance from prior imaging (eg, CT). This may require the patient to be scanned while sitting upright and leaning forward (posterior chest wall); in a lateral decubitus position (lateral chest wall); or semi-supine (anterior chest wall including superior sulcus). Minimising patient movement and the time delay between initial TUS assessment and the subsequent procedure is critical to the success of any intervention from a diagnostic, therapeutic and safety perspective. Still images and, ideally, short video clips should be archived for all ultrasound scans for training, quality and medicolegal purposes. We would encourage clinicians to develop a standardised method of reporting the results of TUS scans for their centre, thereby facilitating consistency and ease of handover between individual practitioners involved in a patient's care from one day to the next.

\section{PATIENT SAFETY}

There is little doubt that concerns about patient safety in pleural interventions have been a major driving force behind the increased uptake of TUS by clinicians other than radiologists over the past decade. The risks associated with even simple thoracentesis have long been recognised, ${ }^{7}$ but it was the UK National Patient Safety Agency alert in $2008^{8}$ which highlighted the need for a change in clinical practice given the extent of avoidable iatrogenic harm caused by 'blind' pleural intervention. Basic use of TUS allows the safe and accurate identification of pleural fluid and other relevant anatomical structures, such as the underlying lung, heart, diaphragm and abdominal viscera. The global evidence that supports the use of TUS as an adjunct to pleural intervention and the associated reduction in risk to patients is overwhelming. ${ }^{9-12}$ Unless resource availability or emergent circumstances present an insurmountable barrier, TUS assessment should be considered an essential 'gold standard' before any pleural intervention for suspected fluid.

However, TUS has the potential to further enhance patient safety beyond the basic determination of whether there is an effusion present or not. The use of TUS does not guarantee appropriate site selection for intervention and might encourage clinicians to venture outside the anatomical safe triangle, since fluid is often most easily seen posteriorly where the costodiaphragmatic recess permits the greatest accumulation and apparent depth of fluid to be seen on TUS with the patient sitting upright. A more posterior approach during pleural intervention increases the risk of injury to the intercostal vessels, owing to increased tortuosity and variable location within the rib space with greater proximity to the spinal midline. ${ }^{13}{ }^{14}$ In turn, this may lead to a life-threatening iatrogenic complication even from an apparently simple small-bore needle aspiration. ${ }^{15} \quad 16$ Published data suggest that TUS can identify the intercostal vessels and positions within an individual rib space using colour
Doppler assessment, ${ }^{17}{ }^{18}$ allowing the operator to select the safest site for subsequent pleural intervention and minimising the likelihood of vascular injury. ${ }^{19}$ Large-scale studies are needed to assess the utility of this technique, given the relative infrequency with which this complication occurs and additional skills required for widespread practice.

The role played by TUS in improving patient safety does not end when the procedure begins. Other than providing the ability to allow real-time guidance of any pleural intervention, TUS also offers the operator an opportunity to identify any iatrogenic complications as early as possible. This allows appropriate treatment to be instituted promptly, limiting the risk of further harm. Postprocedural ultrasound screening at the site of intervention can identify either active bleeding from the parietal pleural surface ${ }^{20}$ or the rapid accumulation of highly echogenic fluid within the pleural space, demonstrating a swirling or gradient effect as heavier cellular material is deposited in the more dependent part of the collection. ${ }^{21}$

A number of studies have also shown that TUS can be used to recognise iatrogenic pneumothorax following procedures such as thoracentesis, transbronchial lung biopsy or image-guided lung biopsy, ${ }^{22-24}$ using key ultrasonographic features such as the presence of a lung point, or absence of B-lines and lung sliding. This last feature is best assessed using standard B-mode ultrasound, but has also been described on M-mode imaging as the 'stratosphere' sign (seen with pneumothorax) as compared with the 'seashore' (normal lung sliding). TUS has some limitations in the diagnosis of pneumothorax; it is largely a binary test (ie, pneumothorax present or not) and has only limited efficacy in determining size through the identification and 'tracking' of a lung point, while its accuracy is questionable in patients with underlying emphysema. ${ }^{25}$ However, the available data suggest that TUS has a sensitivity, specificity and accuracy that may outperform plain chest radiography in the postprocedural setting ${ }^{22-24}$ and has the clear advantage of being immediately available at the bedside. It may also prove to be better than plain chest radiography in ascertaining the subsequent success of any therapeutic intervention that follows, ${ }^{26}$ although this is work that needs to be replicated as part of future research.

\section{NON-EXPANDABLE LUNG}

Non-expandable lung (NEL) is an umbrella term used to describe a situation in which the lung is physically restricted by a pathological process and can no longer fully expand to allow apposition of the visceral and parietal pleural surfaces; this creates a potential space for the recurrent accumulation of pleural fluid. NEL can result from either 'trapped lung' or 'lung entrapment'; terms that sound similar and are frequently used in place of one another, but describe distinct, albeit related, processes. ${ }^{27}$

Pragmatically, the clinical problems that arise are broadly similar whether NEL is caused by proximal bronchial obstruction, a large parenchymal soft tissue lesion, the primary development of fibrous visceral pleural thickening in the absence of an obvious external stimulus (trapped lung) or secondary reactive visceral pleural thickening in the presence of active pleural inflammation, infection or malignancy (lung entrapment). The presence of NEL is likely to make any attempt at pleurodesis futile owing to either a lack of sufficient pleural apposition to allow adhesion formation, or the inherent tendency of the affected lung to recoil from the chest wall. Any effusion associated with NEL is likely to recur quickly as a result of the potential space caused by failure of the lung to expand and take the place of any fluid removed. The symptomatic benefit from draining an effusion secondary to NEL can be highly variable, 
and may even be minimal if the fluid has not caused the mechanics of the chest wall or diaphragm to be impaired. ${ }^{28}$ Indeed, excessive fluid drainage in the context of NEL may worsen a patient's condition as the resultant negative intrapleural pressure can cause distressing symptoms such as chest pain and intractable cough, and result in significant pneumothorax ex vacuo or re-expansion pulmonary oedema.

The end result is a situation in which clinicians have no robust prospective means of predicting which patients with a large effusion have underlying NEL, or indeed which patients with NEL will benefit symptomatically from pleural drainage. Currently available strategies are dependent on the patient having an initial pleural intervention, usually large-volume thoracentesis, to allow either the use of pleural manometry, ${ }^{29}$ and/or the demonstration of pneumothorax ex vacuo on a postdrainage chest radiograph, to confirm the presence of NEL. However, a recent proof of concept study ${ }^{30}$ of the advanced ultrasonographic assessment of patients presenting with pleural effusion has been shown to be potentially useful in identifying the likely presence of NEL before any invasive procedure is necessary (figure 1).

That study ${ }^{30}$ hypothesised that NEL would less readily transmit the cardiac impulse and have more restricted movement within a pleural effusion. In order to prove this, both M-mode ultrasonography and speckle tracking imaging analysis were used, with the aim of measuring tissue displacement and strain pattern (deformation), respectively, within the lung. Pleural manometry was performed during subsequent drainage of the effusion to provide a reference test for NEL using currently available technology; the diagnosis of NEL was then confirmed by consensus evaluation of post-draining imaging studies by two independent physicians. In a study of 81 patients, both speckle tracking (area under receiver operator curve, AUROC 0.86) and M-mode (AUROC 0.79) imaging outperformed pleural manometry (AUROC 0.69 ) in the diagnosis of NEL.

These results ${ }^{30}$ are yet to be replicated in a larger separate study, although subsequent observational data suggest that the same technique can be easily incorporated into everyday clinical practice. ${ }^{31}$ Furthermore, its widespread adoption would require the majority of TUS-competent clinicians to develop skills beyond those currently used in normal everyday practice in the acquisition, analysis and interpretation of TUS findings. There are also no data on how the results might correlate with patientrelated outcomes-notably, symptomatic relief from drainage. However, the ability to diagnose NEL non-invasively and before any pleural intervention, would be a significant step forward. It would allow clinicians to select at an earlier stage the most appropriate management pathway (ie, indwelling pleural catheter as opposed to pleurodesis) and inform patients of the likely outcomes of their treatment.

\section{DIAGNOSTIC TRANSTHORACIC PROCEDURES}

There is a burgeoning evidence to support the use of TUS as an adjunct to a variety of diagnostic transthoracic interventions for chest wall, pleural and lung parenchymal pathology. This requires a clear distinction to be drawn between ultrasound 'assisted' and 'guided' procedures. The former term usually describes a process whereby ultrasound is employed solely to mark out a site for subsequent intervention; as opposed to the latter, where ultrasound is used freehand and in real time to direct the passage of a needle or other device. Both approaches have their place and advantages: ultrasound assistance is less time consuming to learn and perform, whereas real-time guidance facilitates more complex interventions and allows the operator to visually confirm precisely what is being sampled at any given moment. The use of a needle-guide attachment for the ultrasound transducer can further simplify the learning process and performance of guided procedures, allowing accurate sampling to occur with minimal time or fuss.

\section{Pleural biopsy}

The technique of closed pleural biopsy as a means of obtaining diagnostic tissue is long established ${ }^{32} 33$ and has remained popular owing to its accessibility and acceptability to patients and clinicians, particularly as an alternative to thoracoscopy in those areas where healthcare resources are limited. There are good quality data to suggest that the use of image guidance, be that with CT or ultrasound, greatly improves both diagnostic yield and patient safety. ${ }^{34}$ Increasing access to TUS has in turn allowed interventional pulmonologists to successfully deliver this service with outcomes comparable to those of their radiology colleagues ${ }^{35-37}$ (figure 2). TUS has advantages over CT of being non-ionising, quicker to use and capable of allowing the operator to compensate for respiratory movement in real time without the need for breath-hold manoeuvres; however, CT can target pleurally based lesions that might otherwise be inaccessible on TUS (eg, behind ribs or other bony structures). Other factors that may influence diagnostic yield include the underlying pathology (infectious, notably TB vs malignant; and type of malignancy), extent of disease (localised vs diffuse), number of samples taken and operator experience.

There are no robust data to allow a distinction between whether a traditional reverse bevel (eg, Abrams) or core-cutting needle improves sample quality or diagnostic yield. The largest published study dealing with this question ${ }^{35}$ used an ultrasound-assisted technique in 89 patients with suspected tuberculous pleuritis, randomising patients to either Abrams or core-cutting needle biopsies. Although the results implied that Abrams needle biopsy was associated with a better diagnostic yield, the study was criticised for its early cessation, which meant the significance and validity of any findings could be called into question. ${ }^{38}$ The use of an assisted, as opposed to guided, technique may also have had a greater adverse effect on the efficacy of the core-cutting needle biopsies in comparison with the larger Abrams needle. It is worth noting that other physician-based studies of core-cutting needle pleural biopsies $^{36} 37$ that employed a guided technique have reported a higher diagnostic yield $(90.6 \%$ and $84.0 \%$, respectively) than seen in this randomised trial $(78.7 \%))^{35}$

Published data allowing a direct comparison between ultrasound and CT-guided biopsy for pleural disease are limited. A recently published randomised trial, ${ }^{39}$ comparing CT-guided Abrams needle with ultrasound-assisted core-cutting needle biopsy in the investigation of 150 patients with pleural disease, reported superiority of the former technique with a diagnostic sensitivity of $82.4 \%$ vs $66.7 \%(p=0.029)$. However, the decision to use different biopsy needles across the two study arms and an ultrasound-assisted rather than guided technique means that the findings cannot be easily extrapolated to inform everyday clinical practice. A larger retrospective study ${ }^{40}$ compared outcomes from CT and ultrasound-guided core-cutting needle biopsies for both pleural $(\mathrm{n}=86)$ and peripheral lung parenchy$\mathrm{mal}(\mathrm{n}=187)$ lesions, demonstrating no significant difference in diagnostic sensitivity between the two techniques, and that the use of ultrasound was quicker, cheaper and associated with a lower risk of iatrogenic pneumothorax.

On the basis of the data currently available, the authors suggest that TUS can generally be regarded as an appropriate and 

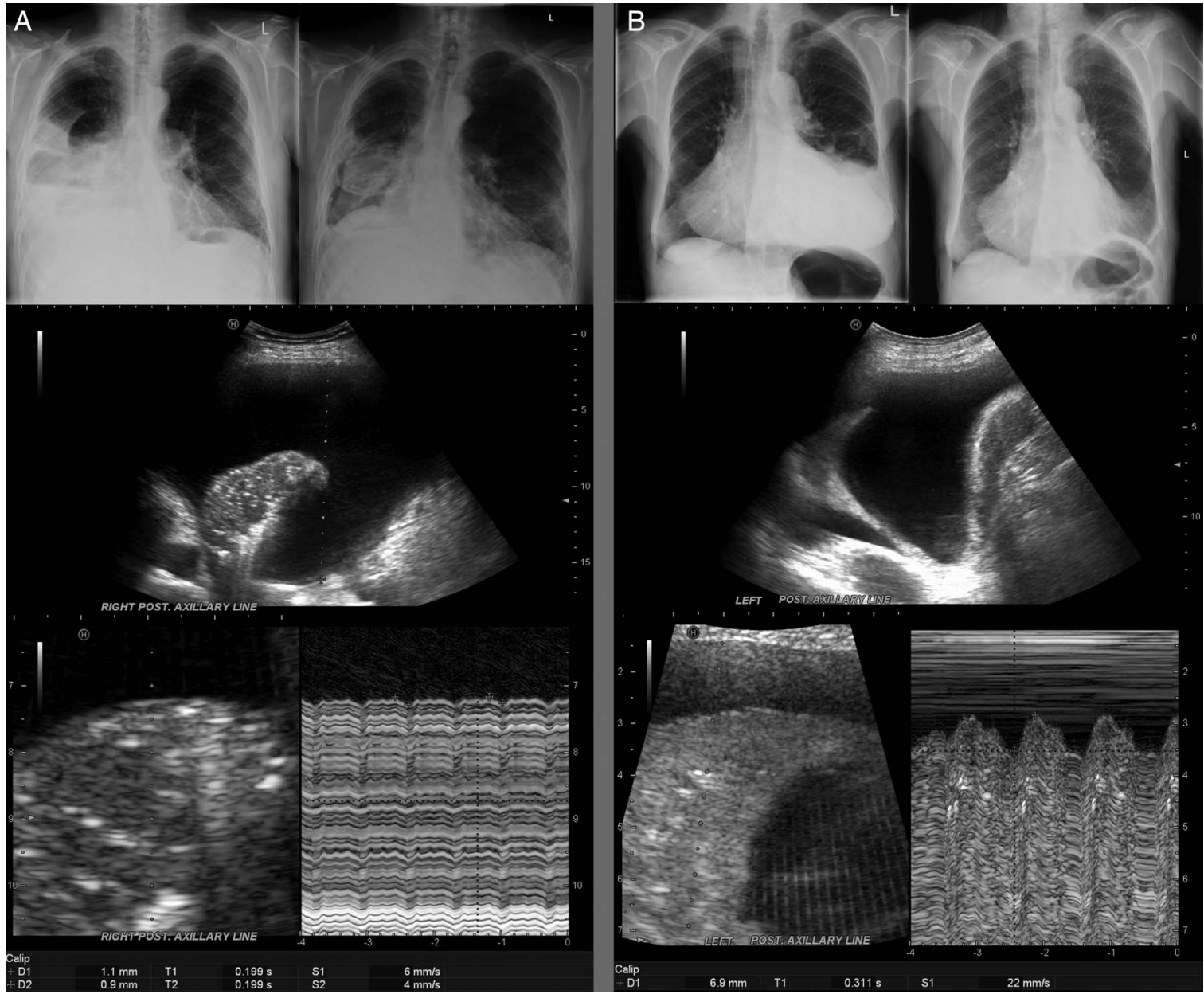

Figure 1 Ultrasonographic assessment of lung movement using M-mode: (A) panel of images showing pre- and post-drainage chest X-ray (CXR) with non-expandable lung; corresponding pre-drainage thoracic ultrasound (TUS) with consolidated, poorly collapsing lung and poor transmission of cardiac impulse; (B) panel of images showing pre- and post-drainage CXR with normally expanding lung; corresponding pre-drainage TUS shows normal atelectatic lung with good transmission of cardiac impulse (note the clear arterial waveform with dicrotic notch).

Figure 2 Thoracic ultrasound (TUS)-guided pleural biopsy: (A) sterile theatre procedural set-up with operator, patient in lateral decubitus position and ultrasound machine in line; (B) close up of ultrasound probe and needle demonstrating real-time in-plane technique; (C) Abrams and core-cutting biopsy needles; (D) TUS image showing core-cutting biopsy needle crossing chest wall and pleural thickening (solid white arrows) with tip of needle visible in small effusion.

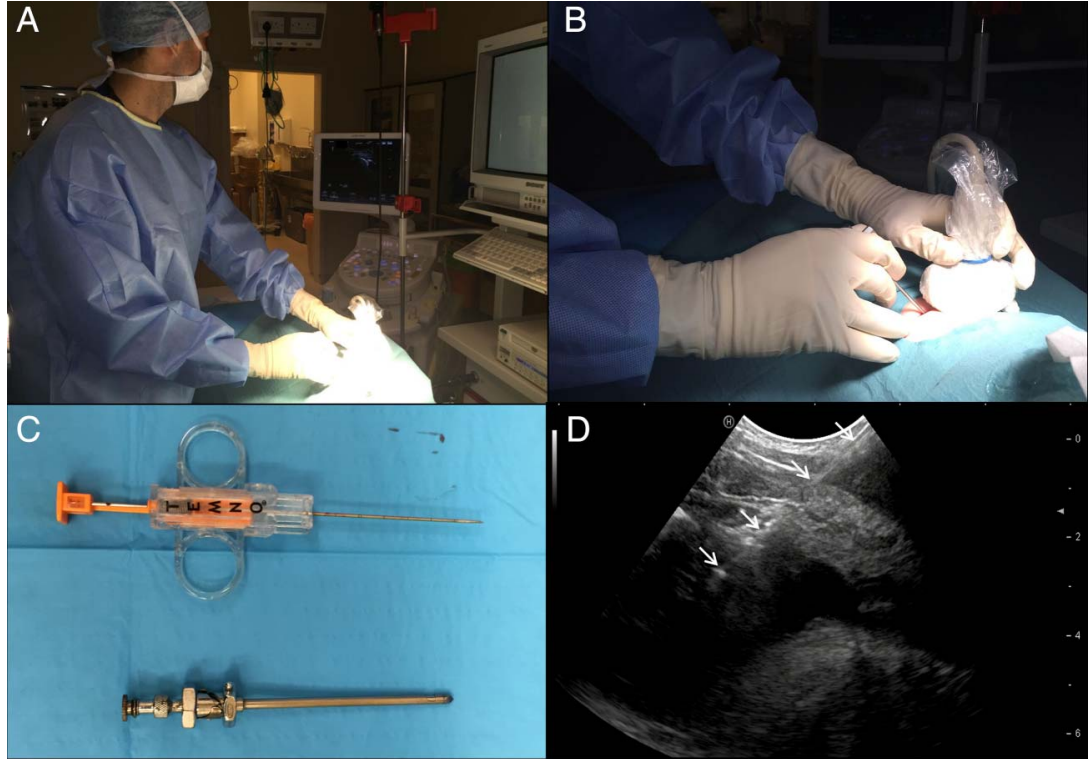

equivalent alternative to CT when performing image-guided pleural biopsies, with the caveat that the operator should be trained in the use of real-time TUS guidance. Between 6 and 10 biopsy specimens should be taken, ideally in different planes of orientation, to ensure adequate sampling. In the absence of any overt pleural abnormalities, favouring a lower (supradiaphramgatic) 
location is more likely to yield a positive diagnosis; ${ }^{41}$ this is probably because metastatic disease favours the lower part of the parietal pleura owing to the distribution of stomata responsible for draining the pleural space into adjacent lymphatics. Pending further comparative studies, clinicians are advised to use the type of biopsy needle that they are most familiar and comfortable with from their everyday practice.

\section{Lung aspirate and biopsy}

Image-guided percutaneous lung sampling techniques have been used for diagnosis in a number of respiratory conditions for over half a century, with the investigation of suspected malignancy being the most widely recognised indication. Although CT-guided biopsy is, by some margin, the most common approach, it is worth noting that TUS-guided sampling of peripheral lung lesions is an equally long-established method, ${ }^{42}$ and may even be advantageous in specific circumstances for reasons similar to those outlined for pleural biopsy. The use of TUS-guided lung biopsy or aspirate for peripheral lung lesions requires appropriate case selection in advance; the mass or nodule must be adjacent to the chest wall with no aerated lung interposed between the two in order to be visualised ultrasonographically (figure 3). The extent of the pleural contact made by the lesion of interest is likely to have a direct influence on diagnostic yield. ${ }^{43}$ Prior CT imaging should be reviewed to simplify and correlate anatomical localisation of the target lesion(s). The majority of lesions will be hypoechoic with clear echogenic demarcation at the interface with surrounding aerated lung; further ultrasonographic assessment may be feasible in larger masses to allow the identification of both necrotic areas, according to echotexture, and highly vascularised areas using colour Doppler. This in turn allows directed sampling under real-time TUS guidance with the intention of maximising diagnostic yield. $^{44}$

The majority of early data on the use of TUS-guided biopsy of lung lesions related to the procedure as performed by specialist radiologists; a recently published literature review and pooled analysis ${ }^{45}$ reported an overall diagnostic accuracy of $88.7 \%$ for the technique, as compared with $92.1 \%$ for CT-guided biopsy. Iatrogenic complications, notably pneumothorax, were less frequent with TUS-guided procedures $(4.4 \%$ vs $20.5 \%$ for CT-guided biopsy), although this may simply be because only lower-risk peripheral lesions were amenable to ultrasound assessment and sampling. Nonetheless, there is a growing body of literature describing the use of TUS-guided lung biopsy by interventional pulmonologists, ${ }^{46} 47$ reflecting the increased access to, and skill with, ultrasound in this group, together with the need to provide a joined-up and efficient diagnostic service for patients with suspected intrathoracic malignancy.

Although less well recognised, TUS-guided lung sampling has a potential diagnostic role outside the sphere of suspected malignancy. Pneumonia is the most common infectious disease worldwide and responsible for a significant and increasing burden of mortality and morbidity across all age groups. ${ }^{48}$ In the majority of cases a causative organism will never be identified, leaving patients and clinicians dependent on 'best guess' broad spectrum antibiotics. The risk of an adverse outcome is greatly increased in those individuals in whom this initial treatment proves ineffective. Furthermore, in an era of increasing bacterial resistance the ability to target therapy and maintain antibiotic stewardship is likely to become ever more important. The technique of transthoracic needle aspiration (TTNA) or 'lung tap' is well described in the literature in both adults and children, ${ }^{49-52}$ with a high microbiological yield of around $50 \%$ and low complication rates of $<5 \%$ (most commonly localised pneumothorax or transient haemoptysis) even for nonradiologically guided sampling. The procedure itself is relatively quick and straightforward, requiring only the insertion of an ultrafine (usually $\leq 22 \mathrm{G}$ ) needle into consolidated lung and subsequent aspiration under aseptic conditions. The growing evidence for the use of TUS as a front-line diagnostic tool for pneumonia in the hospital setting ${ }^{53}{ }^{54}$ naturally lends itself to the concurrent delivery of TTNA to inform subsequent treatment choices. A pilot study assessing the feasibility and safety of TUS-guided TTNA in pneumonia ${ }^{55}$ has demonstrated promising results; further evaluation in a larger prospective study is now needed to determine how this might influence key clinical outcomes.

\section{Lymph node sampling}

The ability to recognise and sample abnormal thoracic and cervical lymphadenopathy that is accessible to ultrasonographic evaluation should be considered a key skill for the interventional pulmonologist and lung cancer physician. This has been recognised by its inclusion in the more advanced levels of some TUS training standards. ${ }^{3}$ It allows the responsible clinician to both diagnose and, in the case of suspected malignancy, potentially stage a patient's disease, with implications for subsequent treatment. ${ }^{56}$ The process of scanning for and, if necessary, sampling lymph nodes is often straightforward enough to be performed in the outpatient setting at the time of a patient's initial clinical evaluation, thereby streamlining the diagnostic pathway and potentially preventing the need for more invasive procedures at a later stage.

In-depth assessment of lymphadenopathy requires a highfrequency $(8-12 \mathrm{MHz})$, usually linear transducer rather than the low-frequency $(2-5 \mathrm{MHz})$ curvilinear probes commonly used for thoracic work. Characterising features, including size, shape, margin, echogenicity and vascularization, all help to distinguish between benign and pathological lymph nodes (figure 4). The former will tend to appear as ovoid or triangular structures with a hypoechoic cortex and hyperechoic medulla, representing the central fatty hilum. In an acute inflammatory response a node may become enlarged, although rarely beyond $2 \mathrm{~cm}$ in diameter, with a large echogenic hilum secondary to the ongoing healing process. Gross enlargement, rounding of shape with loss of the hilum, asymmetrical or nodular cortical thickening, necrotic change, capsular irregularity or disruption and hypervascularity should all be considered features suggesting the possibility of malignant involvement and warranting sampling, either by fine needle aspiration or core-cutting needle biopsy.

Although the ultrasonographic assessment and sampling of cervical and supraclavicular lymphadenopathy is widely acknowledged and used, ${ }^{56}$ it is worth mentioning that a number of mediastinal stations can also be evaluated and accessed transcutaneously under direct ultrasound guidance. ${ }^{57} 58$ However, with the increasing availability of endobronchial and endoscopic ultrasound as alternatives to surgical mediastinoscopy, these newer techniques have become the gold-standard diagnostic investigations in the staging of lung cancer ${ }^{59}$ and rendered TUS somewhat redundant in this setting. Nonetheless, one prospective study ${ }^{60}$ reported a diagnostic success of $93.3 \%$ from a combined ultrasound-assisted fine needle aspirate and cutting needle biopsy procedure in the investigation of patients $(n=45)$ with anterosuperior mediastinal masses. This physician-led study reported no major complications after intervention and precluded the need for a surgical procedure in the majority of 
Figure 3 Non-small cell lung cancer: (A) axial CT image showing right lower lobe mass and adjacent pleural effusion; (B) thoracic ultrasound image in same patient demonstrating simple effusion and underlying tumour within lung tissue, with areas of necrosis and multiple hypoechoic channels consistent with fluid-filled bronchi.

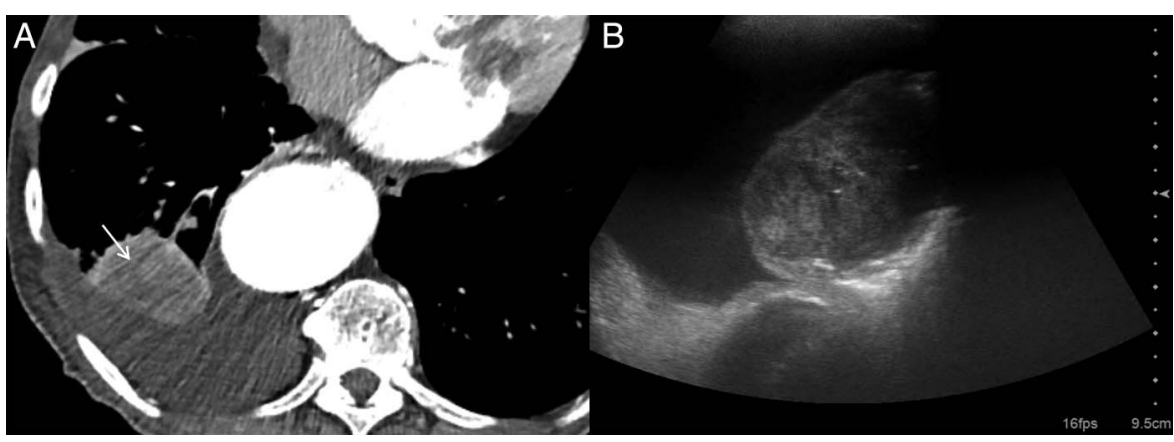

cases; these promising results merit further consideration and evaluation of the technique in an appropriately selected patient population.

\section{Medical thoracoscopy}

Medical thoracoscopy (also described as local anaesthetic thoracoscopy or pleuroscopy) has become a gold standard investigation for the patient with suspected pleural malignancy and/or a unilateral effusion of unknown aetiology. ${ }^{61}$ It provides both diagnostic and therapeutic value, and is widely acknowledged as being a generally safe and well-tolerated procedure. ${ }^{62}$ The majority of medical thoracoscopies will be performed in patients with moderate to large effusions, thereby facilitating easy access to the pleural space as the underlying lung is collapsed and kept clear of the chest wall. However, for more advanced practitioners the use of TUS as an adjunct to the procedure can facilitate safer and more complex intervention in patients with pleural disease but little or no associated fluid, who might previously have required a surgical procedure under general anaesthesia.

Accessing the pleural space in the absence of any fluid generally requires the induction of an artificial pneumothorax in order to create a working area for the thoracoscope. This in turn requires there to be no adhesions present between visceral and parietal pleural surfaces that might otherwise prevent the underlying lung from collapsing. A number of studies ${ }^{63-66}$ have correlated the reduction or absence of normal lung sliding on TUS with the presence of significant pleural adhesions that might either prevent or complicate a thoracoscopic procedure, quoting sensitivities and specificities for the technique of $>80 \%$. This can allow patients to be counselled in advance on the need for a more complex or open surgical intervention that permits dissection apart of the pleural surfaces, or conversion to an alternative diagnostic approach such as image-guided biopsy. $^{36}$

Assuming that pleural adhesions can be successfully excluded, the periprocedural use of real-time TUS can then be used to facilitate the safe induction of an artificial pneumothorax by means of blunt dissection ${ }^{67}$ or Boutin needle introduction ${ }^{68}$ before placement of the trocar and port (figure 5). This allows the operator to directly visualise on TUS the passage of instruments through the chest wall, limiting the risk of injury to the underlying lung; and also to observe the development of a successful pneumothorax through the loss of both lung sliding and

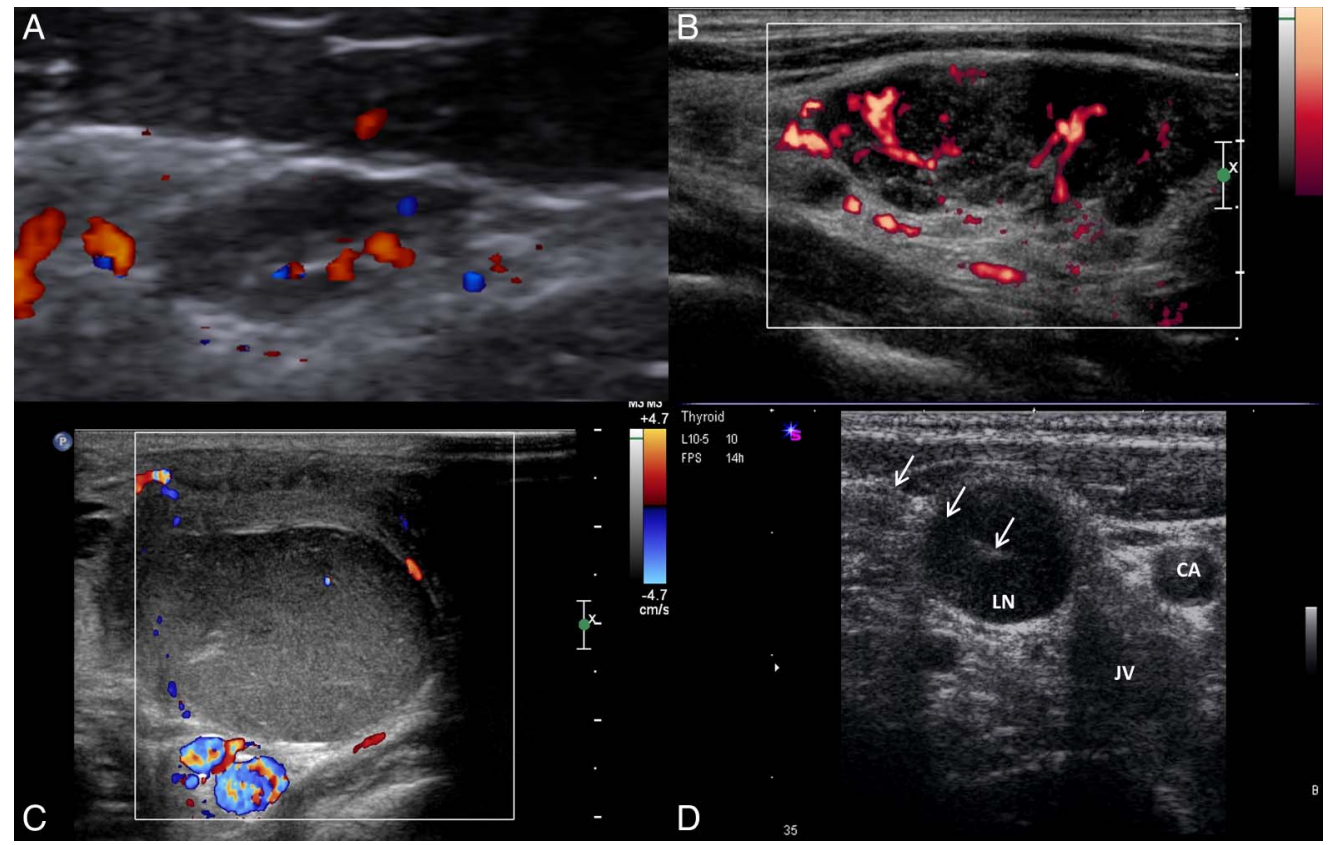

Figure 4 Lymph nodes: (A) reactive lymph node with echogenic fatty hilum and hilar pattern of blood flow; (B) malignant lymph node with loss of hilar architecture and hypervascularity with multiple feeding vessels; (C) necrotic lymph node with rounded, diffusely hypoechoic appearance and largely peripheral vascular pattern; (D) ultrasound-guided fine needle aspiration (solid white arrows) of enlarged cervical lymph node (LN) with adjacent carotid artery (CA) and jugular vein (JV). 
Figure 5 Thoracic ultrasound-guided pneumothorax induction for thoracoscopy: (A) Boutin needle with sharp and blunt trocars; (B) pre-intervention ultrasound showing small effusion and lung adjacent to chest wall in majority of field of view; (C) Boutin needle (solid white arrows) breaching parietal pleura and being introduced into effusion; (D) induction of pneumothorax as air is entrained through open Boutin needle, causing bilateral lung points (solid white arrows) around needle tip.

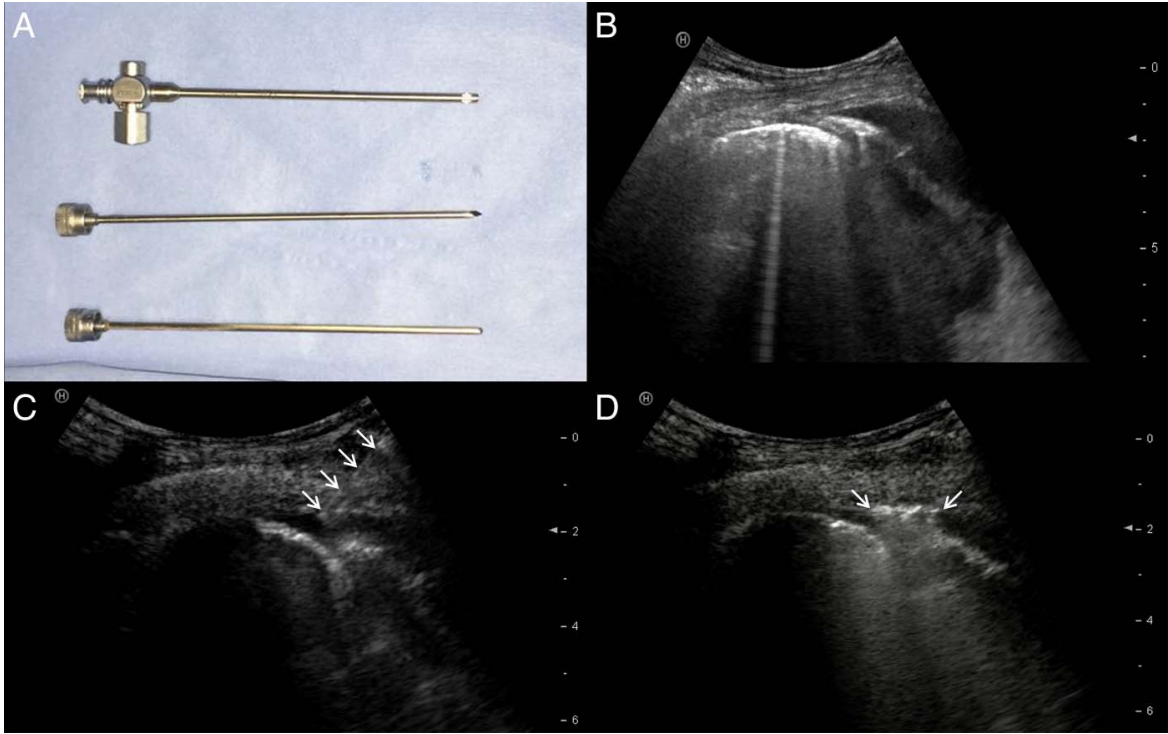

B-lines, alongside the identification of cardinal ultrasonographic features (eg, air-fluid level for hydropneumothorax; stratosphere sign on M-mode assessment for complete pneumothorax).

\section{OUTCOME PREDICTION}

The ability to predict the likely success of any medical intervention at the outset of treatment is crucial to clinicians since it allows patients to be appropriately counselled in advance and, if necessary, contingency plans considered in the event of potential failure. There is a substantial evidence to suggest that findings on TUS for different pleural conditions can give clinicians an insight into a patient's probable response to treatment.

There is considerable variation in the symptomatic benefit of drainage to patients with pleural effusions of all causes, particularly in those cases where cardiac and respiratory morbidities coexist. Very often, clinicians are left with no option but to perform a large-volume thoracentesis in order to assess response and before planning definitive treatment in the event of future recurrence. Although older data suggested that thoracentesis had little impact on either lung volumes or gas exchange, ${ }^{69} 70$ we now know that patients in whom an effusion is associated with flattening, inversion or paradoxical motion of the ipsilateral hemidiaphragm on TUS will almost always experience significant symptomatic benefit from having their fluid drained due to the improvement in respiratory mechanics. ${ }^{71} 72$ This does not always require complete drainage of the effusion, but rather removal of enough fluid to reduce intrapleural pressure and restore the normal anatomical configuration and function of the hemidiaphragm. The identification of inversion or paradoxical movement of the diaphragm in association with an effusion on TUS, should therefore prompt the responsible clinician to consider swift action in the knowledge that drainage will be highly likely to alleviate a patient's dyspnoea, even if the volume of fluid present does not appear to be particularly substantial.

The ultrasonographic identification of septations within a pleural effusion also has the potential to predict the outcome of subsequent clinical treatment. Pleural infection is increasingly common in both adult and paediatric populations, and continues to be associated with significant morbidity and mortality despite apparent diagnostic and therapeutic advances. ${ }^{73}$ Standard initial treatment consists of broad-spectrum antibiotics and percutaneous drainage via tube thoracostomy. ${ }^{74}$ At present there are no robust means of predicting at baseline presentation which patients are likely to have a worse clinical outcome, and might therefore be suitable for escalation of their initial treatment; in particular, the early use of more aggressive strategies, such as surgical clearance or intrapleural fibrinolytic agents. Intuitively, the identification on TUS of either loculation (multiple non-communicating pockets of fluid within the hemithorax) or septation (the formation of internal fibrinous strands within a fluid collection) in an active pleural infection might be expected to result in a more complicated treatment course. A number of retrospective studies in both adults ${ }^{75} 76$ and children ${ }^{77}$ have reported an association of these more complex effusions with poorer outcomes, including the failure of initial medical treatment and percutaneous drainage, an increased need for thoracic surgical intervention and mortality from pleural infection. These data must be interpreted with caution given their retrospective nature, and further prospective studies should examine the question of whether TUS findings in infected pleural collections are a true predictor of key clinical outcomes.

The recently reported TIME3 study ${ }^{78}$ examined the issue of septated malignant pleural effusions and whether the use of intrapleural urokinase had an influence on dyspnoea relief and pleurodesis success. This randomised controlled trial demonstrated that the use of intrapleural fibrinolytic agents had no significant impact on either of the two primary outcome measures, and indeed that these patients $(n=71)$ had a substantial mortality rate of $50 \%$ within 60 days of enrolment. The study authors concluded that patients with septated malignant effusions which do not drain following tube thoracostomy should not be subjected to subsequent intrapleural fibrinolytic therapy, and instead that alternative palliative measures should be started to provide symptomatic relief. ${ }^{78}$

The successful use of TUS to identify the presence of pleural adhesions before thoracoscopic intervention ${ }^{63-66}$ has been discussed earlier in this article. However, the same technique has also been used in small animal ${ }^{79}$ and human ${ }^{80} 81$ studies to predict outcomes in cases of chemical pleurodesis, implying that the use of TUS to confirm the continued presence or absence of lung sliding could predict longer-term treatment success or failure. The majority of patients with malignant pleural effusion continue to be managed with intercostal chest tube drainage and 
subsequent talc slurry pleurodesis, a process that necessitates a 1 -week stay in hospital, on average, and is associated with an approximately $20-30 \%$ failure rate. ${ }^{82}$ Early identification of those patients in whom pleurodesis has, or is likely to have, failed would allow clinicians to expedite the discussion and planning of alternative management strategies such as indwelling pleural catheter insertion, while the ultrasonographic confirmation of adhesion formation might facilitate early hospital discharge of those patients with treatment success. A recently opened randomised controlled trial (ISRCTN 16441661) addressing the role of TUS in predicting pleurodesis success in malignant pleural effusion management will hopefully answer these questions in the near future.

\section{FUTURE DIRECTIONS}

The speed of change that has been seen in the interventional use of TUS in recent years means that training standards have become obsolete in some areas and do not necessarily reflect current practice at either a basic or more advanced level. The inclusion of basic ultrasound training in medical undergraduate curricula is also likely to become commonplace and will further increase the uptake and use of TUS by a wide range of clinicians. ${ }^{83}$ Consequently, there is a growing need for an internationally based expert panel to draw up recommendations that will standardise both training requirements and nomenclature. Curricula that focus on the specific needs of a subspecialty group of clinicians ${ }^{84}$ and use high-fidelity simulation training for interventions ${ }^{85}$ are likely to become more common, as is the incorporation into training programmes of structured assessment tools for competence in both TUS assessment ${ }^{86} 87$ and associated procedures. ${ }^{88} 89$

There are a number of clinical questions relating to TUS that are either already the subject of specific clinical studies or may be answered by other routes in the coming years. The use of TUS to predict key outcomes from a variety of pathologies and therapeutic interventions shows most promise-in particular, whether the baseline presence or subsequent development or resolution of septations in the case of an infected or malignant pleural collection can predict morbidity or mortality; and whether early changes in lung sliding are a prospective marker of longer-term pleurodesis success. Patient-reported outcome measures are of increasing interest and importance, particularly in patients with malignant pleural disease where the majority of interventions are carried out with the aim of achieving symptom control. Specific features on TUS, notably diaphragm function, have been proposed to predict the probability of symptomatic benefit from a subsequent intervention-these merit further assessment in a prospective study. ${ }^{90}$ There has not yet been a robust prospective study of whether TUS or CT-guided biopsy is better for the diagnosis of pleural or indeed peripheral lung parenchymal disease. TUS-guided fine needle aspiration of the lung may also have diagnostic value in parenchymal lung infection and appears to be safe and well-tolerated in small pilot studies; again, a large multicentre trial is needed to determine whether this can change relevant outcomes, such as hospital stay, rate of positive microbiological yield, antibiotic selection and duration.

Research is continuing into ways of overcoming the natural limitations of TUS in the assessment of lung pathology; in particular, for the identification and sampling of peripheral soft tissue lesions. Small-scale feasibility studies using contrast-enhanced ultrasound $^{91-93}$ and tissue elastography ${ }^{94}{ }^{95}$ to characterise subpleural nodules or masses and, if appropriate, sample them for diagnostic purposes, have shown early promise in improving diagnostic accuracy by distinguishing viable from necrotic tissue. However, in the absence of comparative data, it is unclear how these techniques provide additional value. Previous work in this field has looked at the timing, pattern and extent of contrast enhancement to allow TUS to separate out different benign and malignant processes, predominantly in lung parenchymal tissue. ${ }^{96}$ There has been little or no focus on whether contrast-enhanced ultrasound might have an impact on the diagnosis and evaluation of pleural disease; in particular, the recognised problem of patients given a false-negative diagnosis of non-specific pleuritis following thoracoscopic or percutaneous biopsy. ${ }^{97}$ This problem is already being dealt with by a randomised study using PET-CT to guide further pleural biopsies in patients with a negative first sample (ISRCTN 14024829); however, it would be of interest to see whether contrast-enhanced TUS could offer an alternative by identifying similarly vascular or metabolically active tissue and allow an immediate targeted intervention.

The use of second-order analysis of ultrasound imaging, looking at features such as grey-scale texture, is increasingly used in other subspecialty fields but has thus far found limited use within the thorax. ${ }^{98}$ Finding a way of applying this technique in real time during a procedure is likely to be crucial to its success, and will require a software algorithm that allows an ultrasound machine to evaluate a region of interest and inform the operator immediately before diagnostic or therapeutic intervention. Detailed grey-scale analysis is likely to have potential applications throughout the chest; TUS practitioners are already familiar with, for example, the basic use of echogenicity and heterogeneity versus homogeneity to differentiate exudative and transudative pleural fluid. Acquiring a more detailed analysis of grey-scale patterns within different types of pleural fluid, lung and/or pleural lesions may in time facilitate more rapid diagnosis and targeted interventions using TUS. At the preclinical level, there is interest in whether high-intensity focused ultrasound can be used effectively in the lung as a means of safely targeting tumours while limiting damage to normal surrounding tissue. ${ }^{99}$ Further studies are needed to establish the technique's safety and efficacy before pilot clinical studies can be considered.

\section{CONCLUSION}

TUS is being used daily by an increasing number of clinicians as part of routine practice. This is particularly apparent in the field of interventional pulmonology, where access to portable highspecification ultrasound equipment has encouraged practitioners to enhance old ways and develop new ways of working. As a new generation of clinicians who have grown up with ultrasound emerges, the idea that it is little more than a tool for confirming the presence of pleural fluid before intervention will become defunct. Understanding how ultrasound can enhance and inform clinical practice and patient care will be crucial over the coming years, and it is likely that research, training and assessment standards in TUS and how it should be used in the interventional setting will have to evolve rapidly to reflect this changing world.

Contributors The article was conceived by JPC, RT-M, VSN and NMR. JPC was responsible for the initial draft preparation and revision. All authors were involved in reviewing each draft and approving the final manuscript. JPC and NMR are responsible for the overall content as guarantors.

Competing interests None declared.

Provenance and peer review Not commissioned; externally peer reviewed.

\section{REFERENCES}

1 Lichtenstein D, Goldstein I, Mourgeon E, et al. Comparative diagnostic performances of auscultation, chest radiography, and lung ultrasonography in acute respiratory distress syndrome. Anesthesiology 2004;100:9-15. 
2 Inglis AJ, Nalos $\mathrm{M}$, Sue $\mathrm{KH}$, et al. Bedside lung ultrasound, mobile radiography and physical examination: a comparative analysis of diagnostic tools in the critically ill. Crit Care Resusc 2016;18:124.

3 Board of the Faculty of Clinical Radiology. Ultrasound training recommendations for Medical and Surgical Specialties. 2012. http://www.rcr.ac.uk/docs/radiology/pdf/ BFCR(12)17_ultrasound_training.pdf

4 Mayo PH, Beaulieu Y, Doelken P, et al. American College of Chest Physicians/La Societe de Reanimation de Langue Francaise statement on competence in critical care ultrasonography. Chest 2009;135:1050-60.

5 European Federation of Societies for Ultrasound in Medicine and Biology. Minimum training recommendations for the practice of medical ultrasound 2007 [10 February 2012]. http://www.efsumb.org/uploads/mintraining-Feb2006.pdf

6 Volpicelli G, Elbarbary M, Blaivas M, et al. International evidence-based recommendations for point-of-care lung ultrasound. Intensive Care Med 2012:38:577-91.

7 Seneff MG, Corwin RW, Gold LH, et al. Complications associated with thoracocentesis. Chest 1986;90:97-100.

8 Rapid response report: risks of chest drain insertion. National Patient Safety Agency, 2008, NPSA/2008/RRR003. http://www.nrls.npsa.nhs.uk/resources/? entryid45 $=59887$

9 Diacon $\mathrm{AH}$, Brutsche $\mathrm{MH}$, Solèr $\mathrm{M}$. Accuracy of pleural puncture sites: a prospective comparison of clinical examination with ultrasound. Chest 2003;123:436-41.

10 Duncan DR, Morgenthaler TI, Ryu JH, et al. Reducing iatrogenic risk in thoracentesis: establishing best practice via experiential training in a zero-risk environment. Chest 2009;135:1315-20.

11 Mercaldi CJ, Lanes SF. Ultrasound guidance decreases complications and improves the cost of care among patients undergoing thoracentesis and paracentesis. Chest 2013:143:532-8.

12 Gordon CE, Feller-Kopman D, Balk EM, et al. Pneumothorax following thoracentesis: a systematic review and meta-analysis. Arch Intern Med 2010;170:332-9.

13 Helm EJ, Rahman NM, Talakoub 0, et al. Course and variation of the intercostal artery by CT scan. Chest 2013;143:634-9.

14 Dewhurst C, O'Neill S, O'Regan K, et al. Demonstration of the course of the posterior intercostal artery on $\mathrm{CT}$ angiography: relevance to interventional radiology procedures in the chest. Diagn Interv Radiol 2012;18:221-4. doi:10.4261/ 1305-3825.DIR.4366-11.1

15 Psallidas I, Helm EJ, Maskell NA, et al. latrogenic injury to the intercostal artery: aetiology, diagnosis and therapeutic intervention. Thorax 2015;70:802-4.

16 Kanai M, Sekiguchi H. Avoiding vessel laceration in thoracentesis: a role of vascular ultrasound with color Doppler. Chest 2015;147:e5-7.

17 Koyanagi T, Kawaharada N, Kurimoto Y, et al. Examination of intercostal arteries with transthoracic Doppler sonography. Echocardiography 2010;27:17-20.

18 Salamonsen M, Ellis S, Paul E, et al. Thoracic ultrasound demonstrates variable location of the intercostal artery. Respiration 2012;83:323-9.

19 Salamonsen M, Dobeli K, McGrath D, et al. Physician-performed ultrasound can accurately screen for a vulnerable intercostal artery prior to chest drainage procedures. Respirology 2013;18:942-7.

20 Corcoran JP, Psallidas I, Ross CL, et al. Always worth another look? Thoracic ultrasonography before, during, and after pleural intervention. Ann Am Thorac Soc 2016;13:118-21. doi:10.1513/AnnalsATS.201508-559CC

21 Wiesen J, Raman D, Adams J, et al. A patient with lung cancer presenting with respiratory failure and shock. Chest 2013;144:e1-4.

22 Reissig A, Kroegel C. Accuracy of transthoracic sonography in excluding post-interventional pneumothorax and hydropneumothorax. Comparison to chest radiography. Eur J Radiol 2005;53:463-70.

23 Sartori S, Tombesi P, Trevisani L, et al. Accuracy of transthoracic sonography in detection of pneumothorax after sonographically guided lung biopsy: prospective comparison with chest radiography. AJR Am J Roentgenol 2007; 188:37-41.

24 Shostak E, Brylka D, Krepp J, et al. Bedside sonography for detection of postprocedure pneumothorax. J Ultrasound Med 2013;32:1003-9. doi:10.7863/ ultra.32.6.1003

25 Slater A, Goodwin M, Anderson KE, et al. COPD can mimic the appearance of pneumothorax on thoracic ultrasound. Chest 2006;129:545-50.

26 Galbois A, Ait-Oufella H, Baudel JL, et al. Pleural ultrasound compared with chest radiographic detection of pneumothorax resolution after drainage. Chest 2010:138:648-55.

27 Doelken P. Clinical implications of unexpandable lung due to pleural disease. Am J Med Sci 2008:335:21-5.

28 Thomas R, Jenkins S, Eastwood PR, et al. Physiology of breathlessness associated with pleural effusions. Curr Opin Pulm Med 2015;21:338-45.

29 Huggins JT, Doelken P. Pleural manometry. Clin Chest Med 2006;27:229-40.

30 Salamonsen MR, Lo AK, $\mathrm{Ng} \mathrm{AC}$, et al. Novel use of pleural ultrasound can identify malignant entrapped lung prior to effusion drainage. Chest 2014;146:1286-93.

31 Corcoran JP, Talwar A, Hallifax RJ, et al. P2 incorporation of an in-depth thoracic ultrasound assessment into routine pre-procedural evaluation of patients with pleural effusions. Thorax 2016;71:A83-4.
32 Abrams LD. A pleural-biopsy punch. Lancet. 1958;1:30-1.

33 Cope C. New pleural biopsy needle; preliminary study. J Am Med Assoc 1958; 167:1107-8.

34 Rahman NM, Gleeson FV. Image-guided pleural biopsy. Curr Opin Pulm Med 2008; 14:331-6

35 Koegelenberg CF, Bolliger CT, Theron J, et al. Direct comparison of the diagnostic yield of ultrasound-assisted Abrams and Tru-Cut needle biopsies for pleural tuberculosis. Thorax 2010;65:857-62.

36 Hallifax RJ, Corcoran JP, Ahmed A, et al. Physician-based ultrasound-guided biopsy for diagnosing pleural disease. Chest 2014;146:1001-6.

37 Chang DB, Yang PC, Luh KT, et al. Ultrasound-guided pleural biopsy with Tru-Cut needle. Chest 1991;100:1328-33.

38 Rahman NM, Davies RJ. Relearning an old lesson: stopping trials early. Thorax 2010;65:851-3

39 Metintas M, Yildirim H, Kaya T, et al. CT scan-guided Abrams' needle pleural biopsy versus ultrasound-assisted cutting needle pleural biopsy for diagnosis in patients with pleural effusion: a randomized, controlled trial. Respiration 2016;91: 156-63.

40 Sconfienza LM, Mauri G, Grossi F, et al. Pleural and peripheral lung lesions: comparison of US- and CT-guided biopsy. Radiology 2013;266:930-5.

41 Koegelenberg CF, Irusen EM, von Groote-Bidlingmaier F, et al. The utility of ultrasound-guided thoracentesis and pleural biopsy in undiagnosed pleural exudates. Thorax 2015;70:995-7.

42 Chandrasekhar AJ, Reynes CJ, Churchill RJ. Ultrasonically guided percutaneous biopsy of peripheral pulmonary masses. Chest 1976;70:627-30

43 Jeon KN, Bae K, Park MJ, et al. US-guided transthoracic biopsy of peripheral lung lesions: pleural contact length influences diagnostic yield. Acta Radiol 2014:55:295-301.

44 Pan JF, Yang PC, Chang DB, et al. Needle aspiration biopsy of malignant lung masses with necrotic centers. Improved sensitivity with ultrasonic guidance. Chest 1993;103:1452-6.

45 DiBardino DM, Yarmus LB, Semaan RW. Transthoracic needle biopsy of the lung. J Thorac Dis 2015;7(Suppl 4):S304-16

46 Targhetta R, Bourgeois JM, Marty-Double C, et al. Peripheral pulmonary lesions: ultrasonic features and ultrasonically guided fine needle aspiration biopsy. J Ultrasound Med 1993;12:369-74.

47 Meena N, Bartter T. Ultrasound-guided percutaneous needle aspiration by pulmonologists: a study of factors with impact on procedural yield and complications. J Bronchology Interv Pulmonol 2015;22:204-8.

48 Quan TP, Fawcett NJ, Wrightson JM, et al. Increasing burden of community-acquired pneumonia leading to hospitalisation, 1998-2014. Thorax 2016;71:535-42.

49 Vuori-Holopainen E, Peltola H. Reappraisal of lung tap: review of an old method for better etiologic diagnosis of childhood pneumonia. Clin Infect Dis 2001:32:715-26.

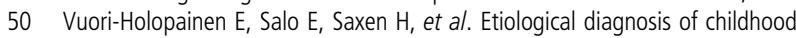
pneumonia by use of transthoracic needle aspiration and modern microbiological methods. Clin Infect Dis 2002;34:583-90.

51 Dorca J, Manresa F, Esteban L, et al. Efficacy, safety, and therapeutic relevance of transthoracic aspiration with ultrathin needle in nonventilated nosocomial pneumonia. Am J Respir Crit Care Med 1995;151:1491-6.

52 Ishida T, Hashimoto T, Arita $\mathrm{M}$, et al. Efficacy of transthoracic needle aspiration in community-acquired pneumonia. Intern Med 2001;40:873-7.

53 Pereda MA, Chavez MA, Hooper-Miele CC, et al. Lung ultrasound for the diagnosis of pneumonia in children: a meta-analysis. Pediatrics 2015;135:714-22.

54 Chavez MA, Shams N, Ellington LE, et al. Lung ultrasound for the diagnosis of pneumonia in adults: a systematic review and meta-analysis. Respir Res 2014:15:50.

55 Wrightson JMC DWM, Rahman NM, Chapman SJ. S42 safety and acceptability of bedside ultrasound-guided transthoracic lung needle aspiration (TLNA) in pneumonia. Thorax 2014;69:A24.

56 Prosch H, Strasser G, Sonka C, et al. Cervical ultrasound (US) and US-guided lymph node biopsy as a routine procedure for staging of lung cancer. Ultraschall Med 2007;28:598-603.

57 Wernecke K, Vassallo P, Pötter R, et al. Mediastinal tumors: sensitivity of detection with sonography compared with CT and radiography. Radiology 1990;175: 137-43.

58 Hirche TO, Wagner TO, Dietrich CF. [Mediastinal ultrasound: technique and possible applications]. Med Klin (Munich) 2002;97:472-9.

59 Silvestri GA, Gonzalez AV, Jantz MA, et al. Methods for staging non-small cell lung cancer: diagnosis and management of lung cancer, 3rd ed: American College of Chest Physicians evidence-based clinical practice guidelines. Chest 2013;143(5 Suppl):e211S-50S.

60 Koegelenberg CF, Diacon AH, Irusen EM, et al. The diagnostic yield and safety of ultrasound-assisted transthoracic biopsy of mediastinal masses. Respiration 2011:81:134-41.

61 Hooper C, Lee YC, Maskell N, Group BTSPG. Investigation of a unilateral pleural effusion in adults: British Thoracic Society Pleural Disease Guideline 2010. Thorax 2010;65(Suppl 2):ii4-17. 
62 Rahman NM, Ali NJ, Brown G, et al. Local anaesthetic thoracoscopy: British Thoracic Society Pleural Disease Guideline 2010. Thorax 2010;65(Suppl 2):ii54-60. doi:10.1136/thx.2010.137018

63 Sasaki M, Kawabe M, Hirai S, et al. Preoperative detection of pleural adhesions by chest ultrasonography. Ann Thorac Surg 2005;80:439-42.

64 Wei $B$, Wang $T$, Jiang $F$, et al. Use of transthoracic ultrasound to predict pleura adhesions: a prospective blinded study. Thorac Cardiovasc Surg 2012;60:101-4.

65 Cassanelli N, Caroli G, Dolci G, et al. Accuracy of transthoracic ultrasound for the detection of pleural adhesions. Eur J Cardiothorac Surg 2012;42:813-18. discussion 8.

66 Medford AR, Agrawal S, Bennett JA, et al. Thoracic ultrasound prior to medical thoracoscopy improves pleural access and predicts fibrous septation. Respirology 2010;15:804-8.

67 Marchetti G, Valsecchi A, Indellicati D, et al. Ultrasound-guided medical thoracoscopy in the absence of pleural effusion. Chest 2015;147:1008-12.

68 Corcoran JP, Psallidas I, Hallifax RJ, et al. Ultrasound-guided pneumothorax induction prior to local anaesthetic thoracoscopy. Thorax 2015;70:906-8.

69 Brown NE, Zamel N, Aberman A. Changes in pulmonary mechanics and gas exchange following thoracocentesis. Chest 1978;74:540-2.

70 Perpina $\mathrm{M}$, Benlloch $\mathrm{E}$, Marco $\mathrm{V}$, et al. Effect of thoracentesis on pulmonary gas exchange. Thorax 1983;38:747-50.

71 Wang JS, Tseng $\mathrm{CH}$. Changes in pulmonary mechanics and gas exchange after thoracentesis on patients with inversion of a hemidiaphragm secondary to large pleural effusion. Chest 1995;107:1610-14.

72 Wang LM, Cherng JM, Wang JS. Improved lung function after thoracocentesis in patients with paradoxical movement of a hemidiaphragm secondary to a large pleural effusion. Respirology 2007;12:719-23.

73 Corcoran JP, Wrightson JM, Belcher E, et al. Pleural infection: past, present, and future directions. Lancet Respir Med 2015:3:563-77.

74 Davies HE, Davies RJ, Davies CW, Group BTSPDG. Management of pleural infection in adults: British Thoracic Society Pleural Disease Guideline 2010. Thorax 2010;65 (Suppl 2):ii41-53.

75 Chen KY, Liaw YS, Wang HC, et al. Sonographic septation: a useful prognostic indicator of acute thoracic empyema. J Ultrasound Med 2000;19:837-43.

76 Chen $\mathrm{CH}$, Chen W, Chen $\mathrm{HJ}$, et al. Transthoracic ultrasonography in predicting the outcome of small-bore catheter drainage in empyemas or complicated parapneumonic effusions. Ultrasound Med Biol 2009;35:1468-74.

77 Ramnath RR, Heller RM, Ben-Ami T, et al. Implications of early sonographic evaluation of parapneumonic effusions in children with pneumonia. Pediatrics 1998;101(Pt 1):68-71.

78 Eleanor KM, Amelia OC, Genevieve W, et al. The Third Therapeutic Intervention in Malignant Effusion Trial (TIME3): a randomised controlled trial to assess dyspnea relief and pleurodesis success following intrapleural urokinase in patients with non-draining malignant pleural effusion. D110 UNUSUAL TUMORS OF THE CHEST. American Thoracic Society International Conference Abstracts: American Thoracic Society; 2016. p. A7937-A.

79 Zhu Z, Donnelly E, Dikensoy 0, et al. Efficacy of ultrasound in the diagnosis of pleurodesis in rabbits. Chest 2005;128:934-9.

80 Leo F, Dellamonica J, Venissac N, et al. Can chest ultrasonography assess pleurodesis after VATS for spontaneous pneumothorax? Eur J Cardiothorac Surg 2005;28:47-9.

81 Alayouty HD, Hasan TM, Alhadad ZA, et al. Mechanical versus chemical pleurodesis for management of primary spontaneous pneumothorax evaluated with thoracic echography. Interact Cardiovasc Thorac Surg 2011;13:475-9.
82 Roberts ME, Neville E, Berrisford RG, et al. Group BTSPDG. Management of a malignant pleural effusion: British Thoracic Society Pleural Disease Guideline 2010. Thorax 2010;65(Suppl 2):ii32-40.

83 Patel SG, Benninger B, Mirjalili SA. Integrating ultrasound into modern medical curricula. Clin Anat 2017;30:452-60.

84 Williamson JP, Twaddell SH, Lee YC, et al. Thoracic ultrasound recognition of competence: a position paper of the Thoracic Society of Australia and New Zealand. Respirology 2017;22:405-8.

85 McSparron Jl, Michaud GC, Gordan PL, et al. Skills-based Working Group of the American Thoracic Society Education Committee. Simulation for skills-based education in pulmonary and critical care medicine. Ann Am Thorac Soc 2015; 12:579-86.

86 Salamonsen M, McGrath D, Steiler G, et al. A new instrument to assess physician skill at thoracic ultrasound, including pleural effusion markup. Chest 2013;144:930-4.

87 Skaarup SH, Laursen CB, Bjerrum AS, et al. Objective and structured assessment of lung ultrasound competence: a multispecialty Delphi consensus and construct validity study. Ann Am Thorac Soc 2017;14, 555-60.

88 Salamonsen MR, Bashirzadeh F, Ritchie AJ, et al. A new instrument to assess physician skill at chest tube insertion: the TUBE-ICOMPT. Thorax 2015;70: 186-8.

89 Shefrin AE, Khazei A, Hung GR, et al. The TACTIC: development and validation of the Tool for Assessing Chest Tube Insertion Competency. CJEM 2015;17:140-7

90 Thomas R, Azzopardi M, Muruganandan S, et al. Protocol of the PLeural Effusion And Symptom Evaluation (PLEASE) study on the pathophysiology of breathlessness in patients with symptomatic pleural effusions. BMJ Open 2016;6:e013213. doi:10. 1136/bmjopen-2016-013213

91 Caremani M, Benci A, Lapini L, et al. Contrast enhanced ultrasonography (CEUS) in peripheral lung lesions: a study of 60 cases. J Ultrasound 2008;11:89-96.

92 Cao BS, Wu JH, Li XL, et al. Sonographically guided transthoracic biopsy of peripheral lung and mediastinal lesions: role of contrast-enhanced sonography. J Ultrasound Med 2011:30:1479-90.

93 Wang J, Zhou D, Xie X, et al. Utility of contrast-enhanced ultrasound with SonoVue in biopsy of small subpleural nodules. Int J Clin Exp Med 2015;8:15991-8.

94 Adamietz BR, Fasching PA, Jud S, et al. Ultrasound elastography of pulmonary lesions - a feasibility study. Ultraschall Med 2014;35:33-7. doi:10.1055/ s-0033-1355893

95 Sperandeo M, Trovato FM, Dimitri L, et al. Lung transthoracic ultrasound elastography imaging and guided biopsies of subpleural cancer: a preliminary report. Acta Radiologica 2015;56:798-805.

96 Görg C, Bert T, Kring R, et al. Transcutaneous contrast enhanced sonography of the chest for evaluation of pleural based pulmonary lesions: experience in 137 patients. Ultraschall Med 2006;27:437-44.

97 Davies HE, Nicholson JE, Rahman NM, et al. Outcome of patients with nonspecific pleuritis/fibrosis on thoracoscopic pleural biopsies. Eur J Cardiothorac Surg 2010;38:472-7.

98 Nguyen P, Bashirzadeh F, Hundloe J, et al. Optical differentiation between malignant and benign lymphadenopathy by grey scale texture analysis of endobronchial ultrasound convex probe images. Chest 2012;141:709-15.

99 Wolfram $\mathrm{F}$, Boltze $\mathrm{C}$, Schubert $\mathrm{H}$, et al. Effect of lung flooding and high-intensity focused ultrasound on lung tumours: an experimental study in an ex vivo human cancer model and simulated in vivo tumours in pigs. Eur J Med Res 2014;19:1. 